\title{
MCMC Method for Exponentiated Lomax Distribution based on Accelerated Life Testing with Type I Censoring
}

\author{
REFAH ALOTAIBI \\ Department of Mathematical Sciences, College of Science, \\ Princess Nourah bint Abdulrahman University, Riyadh, SAUDI ARABIA \\ H. REZK \\ Department of Statistics, Al-Azhar University, Cairo, EGYPT \\ SANKU DEY \\ Department of Statistics, St. Anthony’s College, Shillong, Meghalaya, INDIA
}

\begin{abstract}
Accelerated Life Testing (ALT) is an effective technique which has been used in different fields to obtain more failures in a shorter period of time. It is more economical than traditional reliability testing. In this article, we propose Bayesian inference approach for planning optimal constant stress ALT with Type I censoring. The lifetime of a test unit follows an exponentiated Lomax distribution. Bayes point estimates of the model parameters and credible intervals under uniform and log-normal priors are obtained. Besides, optimum test plan based on constant stress ALT under Type I censoring is developed by minimizing the pre-posterior variance of a specified low percentile of the lifetime distribution at use condition. Gibbs sampling method is used to find the optimal stress with changing time. The performance of the estimation methods is demonstrated for both simulated and real data sets. Results indicate that both the priors and the sample size affect the optimal Bayesian plans. Further, informative priors provide better results than non-informative priors.
\end{abstract}

Key-words:- Accelerated life testing, constant stress, exponentiated Lomax distribution, Bayes estimates, MCMC method, non informative priors, credible intervals, Monte Carlo simulation.

Received: February 8, 2021. Revised: June 24, 2021. Accepted: July 8, 2021. Published: August 1, 2021.

\section{Introduction}

Market competitiveness makes it necessary for companies to produce highly reliable products having longer life time, particularly, electronic devices, computer equipment, missiles, etc. However, for such reliable products/items, it is not easy to obtain enough failure information under normal operating conditions within a specific time period. To overcome such problems, ALT is the most common approach used to ensure rapid failure of products in order to obtain enough failure data or information about life characteristics economically in a short period of time. For greater details, see [1] and [2]. However, in practice, in CSALT the test needs a longer time at low stress levels to yield sufficient failure data.

[1] pointed out that the stress can be applied in different ways such as constant stress ALT (CSALT), step-stress, progressive stress, etc and each stress level has its own advantages and disadvantages. In CSALT, each unit is tested/run at a pre-specified stress level until failure or the test is terminated for any reason. Usually, electronic items like semiconductors, capacitors, etc., run at a constant stress. Due to simplicity in test design and data analysis, CSALTs are frequently adopted in appliance manufacture industries. Conventionally, engineering experience is needed to determine the stress levels of CSALT plans ([3]; [4]). [1] provided excellent review on past developments of CSALT. [5] observed that insufficient failures may cause difficulty in estimating reliability of the product design. In the recent past, CSALT has been studied by many authorsin varied contexts. Readers may refer to the works of [6]; [7]; [8]; [9]; [10]; [11] ; [12] ; [13]; [14] and many others. If the parameters of the model are known and precise, then one can apply maximum likelihood approach for optimal test plan by using the optimization criterion which 
defines as minimization of the asymptotic variance (AV) of the MLE of $t_{p}$ (100pth percentile of the lifetime distribution at the normal stress condition). Using the Delta method and the Fisher information matrix ([15], [16]), AV of the MLE of $t_{p}$ can be obtained. Since the true values parameters are unknown, planning information is usually dependent on uncertainty [17]. Bayesian methods [18] is an alternative for dealing with the uncertainty. The criterion that minimizes the preposterior variance of a quantity of interest determines the optimal test plan which is generally used in Bayesian ALT designs (see, [5]; [19] and [17]). The pre-posterior variance can be computed using Monte Carlo simulation.

This paper presents CSALT model for exponentiated Lomax (ELomax) distribution based on Type I censored failure data. It is assumed that at constant stress level the shape parameter of the distribution follows log linear model. Our aim is to obtain Bayes point estimates and credible intervals under uniform and log-normal priors of the model parameters and to perform sensitivity analysis to investigate how prior distribution and sample size affects the optimal stress changing point. As far as our knowledge goes, no work was carried out to study how prior distribution and sample size affects the optimal stress changing point. We aim to fill up this gap through this work.

The rest of article is organized as follows. In Section 2, we review the ELomax model. In Section 3, we describe the Bayesian approach and Optimization criterion. Simulation study is conductedand findings are listed in Section 4. The importance of ELomax distribution is illustrated by means of aircraft windshield failure time's data set in Section 5. Finally, Section 6 offers some concluding remarks.

\section{The Model}

[20] introduced the ELomax distribution. The ELomax is more flexible than Lomax distribution, for more details see [21]. The cdf, pdf, reliability function and hazard rate function of the ELomax distribution are defined as follows:

$$
\begin{aligned}
& F(t \mid \varphi, \beta, \theta)=\left[1-(1+\varphi t)^{-\beta}\right]^{\theta}, \\
& t, \beta, \theta, \varphi>0, \\
& f(t \mid \varphi, \beta, \theta)=\beta \theta \varphi(1+\varphi t)^{-(\beta+1)}[1- \\
& \left.(1+\varphi t)^{-\beta}\right]^{\theta-1}, t, \beta, \theta, \varphi>0,
\end{aligned}
$$

$$
R(t \mid \varphi, \beta, \theta)=1-\left[1-(1+\varphi t)^{-\beta}\right]^{\theta},
$$$$
t>0 \text {, }
$$

and

$h(t \mid \varphi, \beta, \theta)=\frac{\beta \theta \varphi(1+\varphi t)^{-(\beta+1)}\left[1-(1+\varphi t)^{-\beta}\right]^{\theta-1}}{1-\left[1-(1+\varphi t)^{-\beta}\right]^{\theta}}$,

$t>0$.

where $\theta$ and $\beta$ are the shape parameters and $\varphi$ is a scale parameter of this distribution. When $\varphi=1$, ELomax reduces to the Exponentiated Pareto distribution. Also, when $\varphi=\theta=1$, ELomax reduces to Lomax distribution. The shape of the hazard rate function could be decreasing and inverted bathtub.

\section{Inference based on Type I Censored Samples}

Suppose, in an experiment,there are $r$ levels of high stress $x j, j=1,2, \ldots, r$ and the stress underuse condition is denoted by xuwhere $\mathrm{xu}<\mathrm{x} 1<\mathrm{x} 2<\ldots<\mathrm{xr}$. Using Type I censoring, at each stress level, the experiment terminates once all the items fail or when a fixed censoring time tcj is reached. Assuming that the lifetime at stress level $x j$, tij $, i=1,2, \ldots, n, j=1$, 2, . . . , $\mathrm{r}$, follows ELomax distribution with pdf as given in (2). It is assumed that the stress $\mathrm{xj}$ affects only on the shape parameter $\theta j$ of the ELomax distribution through a log linear model as follows:

$$
\theta_{j}=\exp \left(a+b x_{j}\right), j=1,2, \ldots, r
$$

where $a$ and $b$ are two unknown parameters depending on the nature of the product.

Based on (1) and (2), the likelihood function is given by:

$$
\begin{aligned}
& L(\beta, \varphi, \underline{\theta} \mid \underline{t})=\prod_{j=1}^{r} \prod_{i=1}^{n_{j}}\left[\beta \varphi \theta_{j}(1+\right. \\
& \left.\left.\varphi t_{i j}\right)^{-(\beta+1)}\left[1-\left(1+\varphi t_{i j}\right)^{-\beta}\right]^{\theta-1}\right]^{\delta_{i j}}[1- \\
& \left(1\left[1-(1+\varphi t)^{-\beta}\right]^{\theta}\right]^{1-\delta_{i j}},
\end{aligned}
$$

where $\delta_{i j}$ is an indicator variable such that: 
$\delta_{i j=\{}\left\{\begin{array}{lll}1 & \text { for } & t_{i j} \leq t_{c j} \\ 0 & \text { for } & t_{i j}>t_{c j}\end{array}\right.$,

In our Bayesian analysis, the two sets of prior distributions are assumed and are reported in Table 1. We assume continuous uniform and log-normal prior distributions for the parameters a, b, $\beta$ and $\varphi$ to express our uncertainty in the values of the parameters. Since we do not have prior information, for illustrative purposes we use the following priors:

Table 1. Priors distribution for Bayesian analysis

\begin{tabular}{|c|c|c|c|c|}
\hline Types & $\mathrm{a}$ & $\mathrm{b}$ & $\beta$ & $\varphi$ \\
\hline $\mathrm{P}$ I & $\mathrm{U}(0,6)$ & $\mathrm{U}(0,8)$ & $\mathrm{U}(0,5)$ & $\mathrm{U}(0,7)$ \\
\hline P II & $\begin{array}{c}\text { lnor } \\
(0.001,1000)\end{array}$ & $\begin{array}{c}\text { Inor } \\
(0.001,1000)\end{array}$ & $\begin{array}{c}\text { Inor } \\
(0.001,1000)\end{array}$ & $\begin{array}{c}\text { Inor } \\
(0.001,1000)\end{array}$ \\
\hline
\end{tabular}

where prior PI stands for non-informative prior and considered as, uniform distribution (U). For simplicity, let $\Omega=$ $(a, b, \beta, \varphi)$ and the joint prior for the parameters are considered

$\varepsilon(a, b, \beta, \varphi) \propto 1 / a b \beta \varphi, \sigma_{1} \leq a \leq$

$\sigma_{2}, \sigma_{3} \leq b \leq \sigma_{4}, \sigma_{5} \leq \beta \leq \sigma_{6}, \sigma_{7} \leq \varphi \leq$

$\sigma_{8}$

where $\sigma_{1}, \sigma_{2}, \ldots, \sigma_{8} \quad$ are constants. Assume that the prior PII is informative prior. The informative prior pdf of $\Omega$ follows lognormal (lnor) distribution which are totally uncorrelated with the location and scale parameters $\mu_{1}, \mu_{2}, \mu_{3}, \mu_{4}, \mu_{5}, \mu_{6}, \mu_{7}, \mu_{8}$, and its pdf has the following form

$$
\begin{gathered}
\varepsilon_{1}(a)=\frac{1}{a \mu_{2} \sqrt{2 \pi}} e^{-\left(\frac{\ln (a)-\mu_{1}}{2 \mu_{2}^{2}}\right)^{2}}, a>0, \\
\varepsilon_{2}(b)=\frac{1}{b \mu_{4} \sqrt{2 \pi}} e^{-\left(\frac{\ln (b)-\mu_{3}}{2 \mu_{4}^{2}}\right)^{2}}, b>0, \text { (10) } \\
\varepsilon_{3}(\beta)=\frac{1}{\beta \mu_{6} \sqrt{2 \pi}} e^{-\left(\frac{\ln (\beta)-\mu_{5}}{2 \mu_{6}^{2}}\right)^{2}}, \beta>0,(11) \\
\varepsilon_{4}(\varphi)=\frac{1}{\varphi \mu_{8} \sqrt{2 \pi}} e^{-\left(\frac{\ln (\varphi)-\mu_{7}}{2 \mu_{8}^{2}}\right)^{2}}, \varphi>0
\end{gathered}
$$

The joint informative prior pdf for the parameter $a$, $b, \beta$ and $\varphi$ is provided as

$\Psi(a, b, \beta, \varphi)=$

$\frac{e^{-\left(\frac{\ln (a)-\mu_{1}}{2 \mu_{2}^{2}}+\frac{\ln (b)-\mu_{3}}{2 \mu_{4}^{2}}+\frac{\ln (\beta)-\mu_{5}}{2 \mu_{6}^{2}}+\frac{\ln (\varphi)-\mu_{7}}{2 \mu_{8}^{2}}\right)^{2}}}{a b \beta \varphi \mu_{2} \mu_{4} \mu_{6} \mu_{8}(2 \pi)^{2}}$,

$a, b, \beta, \varphi>0$,

As a result, the posterior pdf with non-informative prior becomes:

$\Psi 1(a, b, \beta, \varphi) \propto \Omega(a, b, \beta, \varphi) L(\mathrm{a}, \beta, \varphi, \underline{\theta} \mid \underline{t})$,

Then

$\Psi 1(a, b, \beta, \varphi) \propto(1 / a b \beta \varphi) \prod_{j=1}^{r} \prod_{i=1}^{n_{j}}\left[\beta \varphi \theta_{j}(1+\right.$ $\left.\left.\varphi t_{i j}\right)^{-(\beta+1)}\left[1-\left(1+\varphi t_{i j}\right)^{-\beta}\right]^{\theta-1}\right]^{\delta_{i j}}[1-$

$\left.\left[1-(1+\varphi t)^{-\beta}\right]^{\theta}\right]^{1-\delta_{i j}}$

Similarly we can write the posterior distribution using informative priors and can obtained Bayes estimators.

Inference on each parameter is based on its marginal posterior density. Thus the marginal posterior density for the parameter $a, b, \beta$ and $\varphi$,respectively,

$f(a \mid t)=\iiint f(\varphi, \beta, \mathrm{b} \mid \underline{t}) d \varphi d \beta d b$,

$f(b \mid t)=\iiint f(\varphi, \mathrm{a}, \beta \mid \underline{t}) d \varphi d a d \beta$,

$f(\beta \mid t)=\iiint f(\varphi, \mathrm{a}, \mathrm{b} \mid \underline{t}) d \varphi d a d b$,

$f(\varphi \mid t)=\iiint f(\mathrm{~b}, \mathrm{a}, \beta \mid \underline{t}) d b d a d \beta$.

Gibbs sampling is used to draw a random sample of the parameters $a, b, \beta$ and $\varphi$ from their own marginal posterior distribution $f(a \mid t), f(b \mid t), f(\beta \mid t)$ and $f(\varphi \mid t)$, respectively, and then estimate the expected value using the sample mean. The squared error loss (SEL ) function is used in order to obtain Bayes estimators from marginal posterior pdf.

\subsection{Bayesian Estimators under the Squared Loss Function}

The posterior mean is a well-known Bayesian 
estimate based on the SEL function. The SEL function is a symmetric loss function with the following formula:

$\operatorname{SEL}(a, \hat{a})=c(\hat{a}-a)^{2}$,

where $\hat{a}$ is an estimator of a and $\mathrm{c}$ is a constant. When the parameters are unknown, the Bayes estimators of $a, b, \beta$ and $\varphi$ under SEL can be obtained as follows:

$$
\begin{aligned}
\hat{a}_{S E L}=E_{1}(a \mid \underline{t}) & =\iiint_{0}^{\infty} c(\hat{a} \\
& -a)^{2} \varepsilon_{1}(a, b, \beta, \varphi) d b d \beta d \varphi, \\
\hat{b}_{S E L}=E_{1}(b \mid \underline{t}) & =\iiint_{0}^{\infty} c(\hat{b} \\
& -b)^{2} \varepsilon_{1}(a, b, \beta, \varphi) d a d \beta d \varphi, \\
\hat{\beta}_{S E L}=E_{1}(\beta \mid \underline{t}) & =\iiint_{0}^{\infty} c(\hat{\beta} \\
& -\beta)^{2} \varepsilon_{1}(a, b, \beta, \varphi) d b d a d \varphi,
\end{aligned}
$$

$\hat{\varphi}_{S E L}=E_{1}(\varphi \mid \underline{t})=\iiint_{0}^{\infty} c(\hat{\varphi}-$

$\varphi)^{2} \varepsilon_{1}(a, b, \beta, \varphi) d b d \beta d a$,

The credible intervals for $a, b, \beta$ and $\varphi$ under SEL can be obtained easily, so the $100(1-\alpha) \%$ CIs for the parameters at $\left(\mathrm{L}_{\mathrm{a}}, \mathrm{U}_{\mathrm{a}}\right),\left(\mathrm{L}_{\mathrm{b}}, \mathrm{U}_{\mathrm{b}}\right),\left(\mathrm{L}_{\beta}, \mathrm{U}_{\beta}\right)$ and $\left(\mathrm{L}_{\varphi}, \mathrm{U}_{\varphi}\right)$, are respectively satisfying

$$
\begin{aligned}
p\left(L_{a} \leq a \leq U_{a}\right) & =1-\alpha \\
= & \int_{L_{a}}^{U_{a}} f(a \mid t) d a,
\end{aligned}
$$

$$
p\left(L_{b} \leq b \leq U_{b}\right)=1-\alpha=
$$

$\int_{L_{b}}^{U_{b}} f(b \mid t) d b$

$p\left(L_{\beta} \leq \beta \leq U_{\beta}\right)=1-\alpha=$

$\int_{L_{\beta}}^{U_{\beta}} f(\beta \mid t) d \beta$,

$p\left(L_{\varphi} \leq \varphi \leq U_{\varphi}\right)=1-\alpha=$

$\int_{L_{\varphi}}^{U_{\varphi}} f(\varphi \mid t) d \varphi$, where the $L$ and $U$ are the lower and upper limit of the interval, respectively.

\subsection{Optimization Criterion}

The posterior mean and posterior variance of $t_{p}$ are given by

$E\left(t_{p}\left(x_{u}\right) \mid \underline{t}\right)=\int_{0}^{\infty} t_{p} f\left(t_{p}\left(x_{u}\right) \mid t\right) d t_{p}\left(x_{u}\right)$,

and

$V\left(t_{p}\left(x_{u}\right) \mid \underline{t)}=\int_{0}^{\infty}\left[t_{p}\left(x_{u}\right)-\right.\right.$

$\left.E\left(t_{p}\left(x_{u}\right)\right)\right]^{2} f\left(t_{p}\left(x_{u}\right) \mid t\right) d t_{p}\left(x_{u}\right)$

respectively. The variance given by (21) cannot be used as an optimization criterion because it depends on the data. However, the preposterior variance of $t_{p}$, which is defined as:

$E\left(V\left(t_{p}\left(x_{u}\right) \mid t\right)\right)=$
$\int_{0}^{\infty} V t_{p}\left(x_{u}\right) f\left(t_{p}\left(x_{u}\right) \mid t\right) d t_{p}\left(x_{u}\right)$.

does not depend on $\underline{t}$ and therefore it will be used as the objective function. The optimal changing time will be the one to minimize $E\left(V\left(t_{p}\left(x_{u}\right) \mid \underline{t}\right)\right)$

where $t_{p}=\frac{1}{\varphi}\left[1-\left(1-p^{\frac{1}{\theta}}\right)^{\frac{-1}{\beta}}\right]$, and $\mathrm{p}$ is the $p^{t h}$ percentile.

\section{Simulation Algorithm}

It's simple to simulate a data vector with

$t_{p}=\frac{1}{\varphi}\left[1-(1-) p^{\frac{1}{\theta_{u}^{\frac{-1}{\beta}}},}\right]_{\text {two-step }}$

process. To begin, the joint prior distribution is used to generate a set of values for the model parameters a, $b, \beta$ and $\varphi$. Second, the conventional inverse transformation method is used to simulate $\mathrm{n}$ i.i.d. failure times from the distribution function provided by (2). The rightcensored observations are simulated failure times that are longer than the censoring time $t_{c}$. The posterior variance $V\left(t_{p} \mid t\right)$ supplied by (21) must be evaluated for each simulated data vector. The Gibbs sampling method is utilized in this work to select a random sample of $t_{p}$ from the 
marginal posterior distribution $f\left(t_{p} \mid t\right)$ and estimate $V\left(t_{p} \mid t\right)$ using the sample variance. The preposterior variance of $t_{p}$ is estimated by averaging $V\left(t_{p} \mid t\right)$ over all simulated data vectors. The preposterior variance of $t_{p}$ is evaluated at discrete points in time to determine the ideal stress changing time of the preposterior variance of $t_{p}$, in order to discover the ideal stress changing point.

\subsection{Simulation}

The following are the simulation steps: The $\mathrm{R}$ language is used to produce accelerated life data from the ELomax distribution at different sample sizes $(20,40)$ at $\varphi=1.2 \beta=1.1, \theta=1.9$. To eliminate posterior dependency on a simulation's starting point, numerous chains with over-dispersed beginning points should be conducted in a single MCMC simulation.

- For three chains, the initial values of $a, b$, $\beta$ and $\varphi$ are as follows: The first chain has beginning values of $\mathrm{a}=0.1, \mathrm{~b}=0.1, \beta=$ 0.1 and $\varphi=0.3$; the second chain has beginning values of $\mathrm{a}=0.3, \mathrm{~b}=0.7, \beta=$ 0.2 and $\varphi=0.4$; and the third chain has beginning values of $\mathrm{a}=0.5, \mathrm{~b}=0.9, \beta=$ 0.3 and $\varphi=0.5$.

- This is a non-formal method of determining convergence. The Monte Carlo standard error (MC error) of the mean is used to calculate the precision of a posterior estimate. According to [22], MC error for each estimate should be less than $5 \%$ of the sample standard deviation (SSD. This rule has been followed in this situation; see Appendix B for more information.

- Assume the experiment ends when all of the items fail or when a specific censoring time $\mathrm{t}$ is reached (Type I censoring). When $\mathrm{n}=\mathrm{n}$ $=10, \mathrm{t}_{\mathrm{c} 1}=15, \mathrm{t}_{\mathrm{c} 2}=7, \mathrm{n}=\mathrm{n}=20, \mathrm{t}_{\mathrm{c} 1}=19, \mathrm{t}_{\mathrm{c} 2}=7$, and $\mathrm{n}=\mathrm{n}=30, \mathrm{t}_{\mathrm{c} 1}=19, \mathrm{t}_{\mathrm{c} 2}=7$.

- It is assumed that values of parameters are known and apply Bayesian method to determine the optimal stress changing point. The objective function is to minimize the asymptotic variance of the $\mathrm{p}^{\text {th }}$ percentile at normal stress, low stress and high stress. Tables 2-5 summarizes the optimal stress changing times $t_{\mathrm{c}}^{*}$ and the corresponding posterior variance of tp at $p=0.1$. The summary of the samples are displayed in
Tables 2-5. It is observed that as sample size increases, accuracy of the estimates improves. Although the stress level saves the experiment time, the results are better in usual conditions. As expected, we observe that the variance of the estimated parameters decreases when we assume informative prior. It is also reasonably to conclude that the interval length is narrower with informative priors. The interval of the parameters a, b, $\beta, \varphi, \theta_{u}, t_{p}\left(x_{u}\right), t_{p}\left(x_{l}\right)$ and $t_{p}\left(x_{h}\right)$, becomes narrower as the sample size increases. Tables 2-5 summarise the results of the samples. As the sample size grows larger, the accuracy of the estimates improves. The results are better in normal conditions, despite the fact that the stress level saves the experiment time. When we assume an informative prior, the variance of the calculated parameters appears to decrease, as expected. With informative priors, it is likewise reasonable to conclude that the interval length is shorter. As the sample size grows, the interval of the parameters a, b, $\beta, \varphi$ $, \theta_{u}, t_{p}\left(x_{u}\right), t_{p}\left(x_{l}\right)$ and $t_{p}\left(x_{h}\right)$, becomes narrower. 
Table 2. Posterior summaries of the model parameter at $\mathrm{n}=20$

\begin{tabular}{|c|c|c|c|c|c|c|c|}
\hline$P$ & Estimate & Mean & $\mathrm{Sd}$ & MC error & median & $95 \% \mathrm{CI}$ & Interval length \\
\hline \multirow{8}{*}{ I } & â & 0.3588 & 0.2067 & 0.00142 & 0.3443 & $(0.0265,0.7994)$ & 0.7729 \\
\hline & $\hat{b}$ & 0.12040 & 0.1013 & $5.011 \mathrm{E}-4$ & 0.09409 & $(0.0037,0.3724)$ & 0.3687 \\
\hline & $\hat{\beta}$ & 3.8210 & 0.827 & 0.00917 & 3.961 & $(2.025,4.953)$ & 2.928 \\
\hline & $\hat{\varphi}$ & 0.0511 & 0.03035 & $3.916 \mathrm{E}-4$ & 0.043 & $(0.0258,0.1213)$ & 0.0955 \\
\hline & $\hat{\theta}_{u}$ & 1.5490 & 0.3146 & 0.002336 & 1.495 & $(1.115,2.297)$ & 1.1820 \\
\hline & $\hat{\mathrm{t}}_{p}\left(x_{u}\right)$ & 1.6600 & 0.3365 & 0.001105 & 1.639 & $(1.065,2.3710)$ & 1.3060 \\
\hline & $\hat{\mathrm{t}}_{p}\left(x_{l}\right)$ & 1.8360 & 0.3782 & 0.00101 & 1.819 & $(1.149,2.6260)$ & 1.4770 \\
\hline & $\hat{\mathrm{t}}_{p}\left(x_{h}\right)$ & 1.5011 & 0.3508 & 0.001473 & 1.47 & $(0.9223,2.2590)$ & 1.3367 \\
\hline \multirow{8}{*}{ II } & â & 0.9607 & 0.02867 & $7.598 \mathrm{E}-5$ & 0.9603 & $(0.9057,1.0180)$ & 0.1123 \\
\hline & $\hat{b}$ & 0.9421 & 0.02744 & 7.369E-5 & 0.9417 & $(0.8893,0.9971)$ & 0.1078 \\
\hline & $\hat{\beta}$ & 1.0660 & 0.03015 & $8.116 \mathrm{E}-5$ & 1.065 & $(1.008,1.126)$ & 0.1180 \\
\hline & $\hat{\varphi}$ & 1.0340 & 0.0319 & $8.778 \mathrm{E}-5$ & 1.033 & $(0.9727,1.097)$ & 0.1243 \\
\hline & $\hat{\theta}_{u}$ & 4.1880 & 0.1289 & $3.345 \mathrm{E}-4$ & 4.185 & $(3.945,4.4500)$ & 0.505 \\
\hline & $\hat{\mathrm{t}}_{p}\left(x_{u}\right)$ & 2.1240 & 0.1322 & $3.131 \mathrm{E}-4$ & 2.119 & $(1.877,2.3950)$ & 0.5180 \\
\hline & $\hat{\mathrm{t}}_{p}\left(x_{l}\right)$ & 3.5770 & 0.2548 & $6.099 \mathrm{E}-4$ & 3.566 & $(3.1050,4.1050)$ & 1.000 \\
\hline & $\hat{\mathrm{t}}_{p}\left(x_{h}\right)$ & 1.2040 & 0.06838 & $1.623 \mathrm{E}-4$ & 1.203 & $(1.076,1.3450)$ & 0.2690 \\
\hline
\end{tabular}


Table 3. Optimum Bayesian design under Type I censoring at $n=20$

\begin{tabular}{|c|c|c|c|c|c|}
\hline \multirow{2}{*}{$\mathrm{P}$} & stress & $\mathrm{E}\left(\operatorname{var}\left(\mathrm{t}_{p} \mid \underline{t}\right)\right)$ & $\mathrm{t}_{c 1}^{*}$ & $\mathrm{t}_{c 2}^{*}$ \\
\hline \multirow{3}{*}{$\mathrm{n}$} & $\mathrm{I}$ & $\mathrm{x}_{u}$ & 0.1925 & 3.6575 & 1.3475 \\
\cline { 3 - 6 } & $\mathrm{x}_{l}$ & 0.2324 & 4.4156 & 1.6268 \\
\cline { 3 - 6 } & $\mathrm{x}_{h}$ & 0.2846 & 5.4075 & 1.9922 \\
\cline { 3 - 6 } & & $\mathrm{x}_{u}$ & 0.07556 & 1.4383 & 0.5293 \\
\cline { 3 - 6 } & $\mathrm{II}$ & $\mathrm{x}_{l}$ & 0.1573 & 2.9887 & 1.1011 \\
\cline { 3 - 6 } & & $\mathrm{x}_{h}$ & 0.3158 & 6.0002 & 2.2106 \\
\hline
\end{tabular}

Table 4. Posterior summaries of model parameters at $n=40$

\begin{tabular}{|c|c|c|c|c|c|c|c|}
\hline $\mathrm{P}$ & Estimate & Mean & $\mathrm{Sd}$ & MC error & median & $95 \% \mathrm{CI}$ & Interval length \\
\hline \multirow{11}{*}{ I } & â & 0.1409 & 0.1117 & $7.427 \mathrm{E}-4$ & 0.1155 & $0.0047,0.4103)$ & 0.4056 \\
\hline & $\hat{b}$ & 0.1786 & 0.1064 & $8.383 \mathrm{E}-4$ & 0.1695 & $(0.0117,0.4076)$ & 0.3959 \\
\hline & $\hat{\beta}$ & 3.691 & 0.8279 & 0.01215 & 3.764 & $(2.0360,4.9400)$ & 2.9040 \\
\hline & $\hat{\varphi}$ & 0.03417 & 0.01657 & $2.636 \mathrm{E}-4$ & 0.02919 & $(0.0181,0.0786)$ & 0.0605 \\
\hline & $\hat{\theta}_{u}$ & 1.265 & 0.1313 & 0.001108 & 1.24 & $(1.0800,1.5870)$ & 0.5070 \\
\hline & $\hat{\mathrm{t}}_{p}\left(x_{u}\right)$ & 1.903 & 0.2525 & $8.067 \mathrm{E}-4$ & 1.889 & $(1.4470,2.4330)$ & 0.9860 \\
\hline & $\hat{\mathrm{t}}_{p}\left(x_{l}\right)$ & 2.245 & 0.3598 & 0.00123 & 2.23 & $(1.5890,2.9870)$ & 1.3980 \\
\hline & $\hat{\mathrm{t}}_{p}\left(x_{h}\right)$ & 1.603 & 0.2675 & 0.001632 & 1.566 & $(1.1760,2.2190)$ & 1.0430 \\
\hline & â & 0.9475 & 0.0278 & 7.621E-5 & 0.9472 & $(0.894,1.003)$ & 0.1090 \\
\hline & $\hat{b}$ & 0.9317 & 0.02618 & 7.47E-5 & 0.9315 & $(0.8813,0.9836)$ & 0.1023 \\
\hline & $\hat{\beta}$ & 1.073 & 0.02712 & $7.744 \mathrm{E}-5$ & 1.072 & $(1.0200,1.1270)$ & 0.1070 \\
\hline
\end{tabular}




\begin{tabular}{|c|c|l|l|l|l|l|l|}
\hline \multicolumn{1}{|c|}{$\mathrm{II}$} & 1.042 & 0.03185 & $9.015 \mathrm{E}-5$ & 1.042 & $(0.9813,1.106)$ & 0.1247 \\
\hline & $\hat{\theta}_{u}$ & 4.112 & 0.1204 & $3.399 \mathrm{E}-4$ & 4.108 & $(3.884,4.356)$ & 0.4720 \\
\cline { 2 - 8 } & $\hat{\mathrm{t}}_{p}\left(x_{u}\right)$ & 2.025 & 0.104 & $2.179 \mathrm{E}-4$ & 2.022 & $(1.8300,2.2380)$ & 0.4080 \\
\hline & $\hat{\mathrm{t}}_{p}\left(x_{l}\right)$ & 3.391 & 0.1976 & $4.162 \mathrm{E}-4$ & 3.385 & $(3.0220,3.7960)$ & 0.7740 \\
\hline & $\hat{\mathrm{t}}_{p}\left(x_{h}\right)$ & 1.155 & 0.05573 & $1.215 \mathrm{E}-4$ & 1.153 & $(1.0500,1.2680)$ & 0.2180 \\
\hline
\end{tabular}

Table 5. Optimum Bayesian design under Type I censoring at $n=40$

\begin{tabular}{|c|c|c|c|c|c|}
\hline \multirow{7}{*}{$\mathrm{n}$} & $\mathrm{P}$ & stress & $\mathrm{E}\left(\operatorname{var}\left(\mathrm{t}_{p} \mid \underline{t}\right)\right)$ & $\mathrm{t}_{c 1}^{*}$ & $\mathrm{t}_{c 2}^{*}$ \\
\hline & \multirow{3}{*}{ I } & $\mathrm{x}_{u}$ & 0.1192 & 1.192 & 2.384 \\
\hline & & $\mathrm{x}_{l}$ & 0.1662 & 1.662 & 3.324 \\
\hline & & $\mathrm{x}_{h}$ & 0.2333 & 2.333 & 4.666 \\
\hline & \multirow{3}{*}{ II } & $\mathrm{x}_{u}$ & 0.0603 & 0.603 & 1.206 \\
\hline & & $\mathrm{x}_{l}$ & 0.1856 & 1.856 & 3.712 \\
\hline & & $\mathrm{x}_{h}$ & 0.5206 & 5.206 & 10.412 \\
\hline
\end{tabular}




\section{Application}

In this section, for illustration purpose, we analyse one real data set which represents the failure times of 84 Aircraft windshield. This data set was first discussed by [23]. These data were recently studied by [24] and [25]. The data set is divided into two samples, $\mathrm{n}_{1}$ and $\mathrm{n}_{2}$ where $\left(\mathrm{n}_{1}+\mathrm{n}_{2}=84\right)$. $\mathrm{n}_{1}$ units have failed during the interval $\left(0, \mathrm{t}_{\mathrm{c}}\right)$ and $\mathrm{n}_{2}\left(84-\mathrm{n}_{1}\right)$ units are still active, where $a, b, \beta, \varphi$ are the population parameters. For achieving the convergence, we apply three chains of the BrooksGelman-Rubin statistics for a given parameter. The summary of the real data set with respect to the unknown parameters $a, \mathrm{~b}, \beta, \varphi, \theta_{u}, t_{p}\left(x_{u}\right), t_{p}\left(x_{l}\right)$ and $t_{p}\left(x_{h}\right)$, where $\mathrm{p}=0.1$, are displayed in Table 6.

- Assume that the experiment is terminated once all the items fail or when a fixed censoring time $t_{c j}$ is reached (Type I censoring) . $\mathrm{t}_{\mathrm{c} 1}=2.49$ and $\mathrm{t}_{\mathrm{c} 2}=3.69$ at $n_{1}$ and $n_{2}$, respectively.

- From Table 7 ,it is observed that the MC error for each estimate is less than $5 \%$ of the $\mathrm{SSD}$, thus the rule of MC error has been achieved. Also, to check the convergence, Gelman-Rubin convergence statistic, $\mathrm{R}$, is applied.

- For WinBUGS simulation convergence, R should be one, or close to one.

- The two-sided $95 \%$ credible intervals for the estimates of parameters $\theta_{u}, \mathrm{t}^{p}\left(\mathrm{x}^{u}\right), \mathrm{t}^{p}$ $\left(\mathrm{x}^{l}\right)$ and $\mathrm{t}^{p}\left(\mathrm{x}^{h}\right)$ of ELomax distribution are reported in Table 6 . As the sample size increases, length of the interval becomes narrower.

- It appears that in case of informative prior, the variance of estimated parameters decreases. 
Table 6. Posterior statistics of model parameters under Type I censoring

\begin{tabular}{|c|c|c|c|c|c|c|c|}
\hline $\mathrm{P}$ & Estimate & Mean & $\mathrm{Sd}$ & MC error & median & $95 \% \mathrm{CI}$ & Interval length \\
\hline \multirow{8}{*}{ I } & $\hat{a}$ & 0.4155 & 0.2634 & 0.004032 & 0.3840 & $(0.0245,0.9966)$ & 0.9721 \\
\hline & $\hat{b}$ & 0.9960 & 0.1998 & 0.003059 & 1.0190 & $(0.5565,1.3100)$ & 0.7535 \\
\hline & $\hat{\beta}$ & 0.9950 & 0.0049 & $2.102 \mathrm{E}-5$ & 0.9966 & $(0.9819,0.9999)$ & 0.0180 \\
\hline & $\hat{\varphi}$ & 1.9110 & 0.0828 & $3.600 \mathrm{E}-4$ & 1.9350 & $(1.6900,1.9980)$ & 0.3080 \\
\hline & $\hat{\theta}_{u}$ & 2.5300 & 0.4535 & 0.006768 & 2.4450 & $(1.9260,3.616)$ & 1.6900 \\
\hline & $\hat{\mathrm{t}}_{p}\left(x_{u}\right)$ & 0.3560 & 0.0781 & 0.001457 & 0.3370 & $(0.2300,0.5890)$ & 0.3590 \\
\hline & $\hat{\mathrm{t}}_{p}\left(x_{l}\right)$ & 0.7041 & 0.0963 & $9.33 \mathrm{E}-4$ & 0.6960 & $(0.5754,0.8769)$ & 0.3015 \\
\hline & $\hat{\mathrm{t}}_{p}\left(x_{h}\right)$ & 1.3040 & 0.1165 & $8.598 \mathrm{E}-4$ & 1.3010 & $(1.0830,1.5390)$ & 0.4560 \\
\hline \multirow{8}{*}{ II } & $\hat{a}$ & 0.9351 & 0.0276 & 7.934E-5 & 0.9347 & $(0.8824,0.9904)$ & 0.1080 \\
\hline & $\hat{b}$ & 0.9233 & 0.02633 & $8.242 \mathrm{E}-5$ & 0.923 & $(0.8726,0.9758)$ & 0.1032 \\
\hline & $\hat{\beta}$ & 1.411 & 0.03529 & $1.212 \mathrm{E}-4$ & 1.41 & $(1.342,1.481)$ & 0.1395 \\
\hline & $\hat{\varphi}$ & 1.126 & 0.03286 & $1.002 \mathrm{E}-4$ & 1.126 & $(1.063,1.192)$ & 0.1290 \\
\hline & $\hat{\theta}_{u}$ & 4.044 & 0.1193 & 3.484E-4 & 4.041 & $(3.819,4.287)$ & 0.4680 \\
\hline & $\hat{\mathrm{t}}_{p}\left(x_{u}\right)$ & 0.7171 & 0.02729 & $5.838 \mathrm{E}-5$ & 0.7165 & $(1.7200,2.031)$ & 1.3110 \\
\hline & $\hat{\mathrm{t}}_{p}\left(x_{l}\right)$ & 1.191 & 0.0458 & $8.642 \mathrm{E}-5$ & 1.19 & $(1.283,1.1030)$ & 0.1800 \\
\hline & $\hat{\mathrm{t}}_{p}\left(x_{h}\right)$ & 1.87 & 0.07924 & $1.504 \mathrm{E}-4$ & 1.869 & $(2.031,1.72)$ & 0.3110 \\
\hline
\end{tabular}


Table7. Optimum Bayesian design under Type I censoring

\begin{tabular}{|c|c|c|c|c|}
\hline \multirow{2}{*}{$\mathrm{P}$} & stress & $\mathrm{E}\left(\operatorname{var}\left(\mathrm{t}_{p}\right) \mid \underline{t}\right)$ & $\mathrm{t}_{c 1}^{*}$ & $\mathrm{t}_{c 2}^{*}$ \\
\hline \multirow{1}{*}{$\mathrm{II}$} & $\mathrm{x}_{u}$ & 0.0026 & 0.0065 & 0.0182 \\
\cline { 2 - 5 } & $\mathrm{x}_{l}$ & 0.0096 & 0.0239 & 0.0672 \\
\cline { 2 - 5 } & $\mathrm{x}_{h}$ & 0.0329 & 0.0819 & 0.2303 \\
\cline { 2 - 5 } & $\mathrm{x}_{u}$ & 0.0099 & 0.0247 & 0.0365 \\
\cline { 2 - 5 } & & 0.0273 & 0.0679 & 0.1007 \\
\hline & $\mathrm{x}_{l}$ & & 0.1676 & 0.2483 \\
\cline { 2 - 5 } & & & & \\
\hline
\end{tabular}

\section{Conclusion}

In this study, we present Bayesian analysis for the type I censored data under CSALT for the exponentiated Lomax distribution usinglog-linear life-stress function. We have investigated how prior distribution and sample size affects the optimal stress changing point. We observe that the variance of the estimated parameters decreases when we assume informative prior. We also observe that length of the credible intervals for the parameters of interest becomes narrower as the sample size increases. Similar results echoed in the real data analysis.It would be of great interest for statistician/ reliability engineers to study the different classical methods of estimation under CSALT for the exponentiated Lomax distribution and log linear acceleration model based on type II, progressive type II censoring data. The work in this direction is in progress and will be reported later. Although we have considered log-linear acceleration model, however, there are several other models where it can be applied.

\section{Acknowledgements:}

The authors thank the Editor and the three referees for their helpful comments aided in improving this article. This research was funded by the Deanship of Scientific Research at Princess Nourah bint Abdulrahman University through the Fast-track Research Funding Program.
Funding: This research was funded by the Deanship of Scientific Research at Princess Nourah bint Abdulrahman University through the Fast-track Research Funding Program.

\section{References:}

[1] W. B. Nelson, Accelerated testing: statistical models, test plans and data analysis 344. Wiley-Inter. science, 2009.

[2] V. Bagdonavicius, M. Nikulin. Accelerated Life Models: Modeling and Statistical Analysis. Chapman and Hall/CRC Press, Boca Raton, Florida,2002.

[3] W. Q. Meeker, L. A, Escobar, Statistical Methods for Reliability Data. New York: Wiley, 1998.

[4] G. B, Yang, Life Cycle Reliability Engineering. Hoboken, NJ: John Wiley \& Sons, Inc., 2007.

[5] X. Liu, L.C.Tang, Planning sequential constant-stress accelerated life tests with stepwise loaded auxiliary acceleration factor, J.Stat.Plann. Inference 140(7) (2010b) 19681985.

[6] L. C. Tang, T. N. Goh, Y. S. Sun, and H. L. Ong, Planning accelerated life tests for censored two-parameter exponential distributions, Naval Research Logistics (NRL) 46(2) (1999) 169-186.

[7] A. J. Watkins, A. M. John, On constant stress accelerated life tests terminated by type II censoring at one of the stress levels, Journal of Statistical Planning and Inference 138(3) (2008) 768-786. 
[8] H. Ma, W. Q. Meeker, Strategy for planning accelerated life tests with small sample sizes, IEEE Transactions on Reliability 59(4) (2010) 610-619.

[9] Abdel Ghaly A, Aly H, Salah R. Different Estimation Methods for Constant Stress Accelerated Life Test under the Family of the Exponentiated Distributions. Quality and Reliability Engineering International 2016; 32(3): 1095-1108.

[10] Nassar M, Dey S. Different estimation methods for exponentiated Rayleigh distribution under constant-stress accelerated life test. Qual Reliab Engng Int 2018: DOI: 10.1002/qre.2349.

[11] Dey S, Nassar M. Classical methods of estimation on constant stress accelerated life tests under exponentiated Lindley distribution. J Appl Stat 2020; 47(6): 975-996.

[12] Dey S, Nassar M. Generalized inverted exponential distribution under constant stress accelerated life test: Different estimation methods with application. QualReliab Engng Int 2020; 36(4): 1296-1312.

[13] H. M. Aly, S. O. Bleed, H. Z. Muhammed. Inference and Optimal Design of Accelerated Life Test using Geometric Process for Generalized Half-Logistic Distribution under Progressive Type-II Censoring . Journal of Data Science, 18(2) (2020). 354 -371.

[14] A. M. Abd El-Raheem, Ehab M. Almetwally, M. S. Mohamed , E. H. Hafez. Accelerated life tests for modified Kies exponential lifetime distribution: binomial removal, transformers turn insulation application and numerical results. AIMS Mathematics, 2021, 6(5), 2021, 5222-5255.

[15] N. Fard, C. Li, Optimal Simple Step Stress Accelerated Life Test Design for Reliability Prediction, Journal of Statistical Planning and Inference 139 (2009) 1799-1808.

[16] E. A. Elsayed, H. Zhang, Design of Optimum Simple Step-Stress Accelerated Life Testing Plans, In Recent Advances in Stochastic Operations Research, T. Dohi, S. Osaki, K. Sawaki, Eds, (2007) 23-38.

[17] T. Yuan, X. Liu, W. and Kuo, Planning simple step-stress accelerated life tests using Bayesian methods, IEEE Trans Reliability 61(1) (2011) 254-263.

[18] A. Pasanisi , M. Keller, E. Parent, Estimation of a quantity of interest in uncertainty analysis: some help from Bayesian decision theory, Reliability EngSystSaf., 100 (2012) 93-101.
[19] Y. Zhang, W. Q. Meeker, Bayesian optimum planning for accelerated life tests, Technometrics 48 (2006) 49-60.

[20] I. B. Abdul-Moniem and H. F. Abdel-Hameed, On Exponentiated Lomax distribution, International Journal of Mathematical Archive 3(5) ( 2012), 2144-2150.

[21] H. Salem, The exponentiated Lomax distribution: different estimation methods, American Journal of Applied Mathematics and Statistics 2 (2014) 364-368.

[22] D. Spiegelhalter, A. Thomas, N. Best D. Lunn, WinBUGS Version 1.4 User Manual. MRC Biostatistics Unit, Cambridge, 2003

[23] D. Murthy, M. Xie, R. Jiang, Weibull models, John Wiley and Sonc, Inc. 2004

[24] M. W. A. Ramos, P. R. D. Marinho, R. V. da Silva and G. M. Cordeiro, The exponentiated Lomax Poisson distribution with an application to lifetime data, Advances and Applications in Statistics 34 (2013) 107-135.

[25] A. H. El-Bassiouny, N. F. Abdo, H. S. Shahen, (2015). Exponential Lomax Distribution.International Journal of Computer Applications 121(13):24-29.

\section{Contribution of individual authors to the creation of a scientific article (ghostwriting policy)}

Refah Alotaibi and H. Rezk carried out the methodology, simulation and the optimization.

Sanku Dey was responsible for editing and review.

\section{Sources of funding for research presented in a scientific article or scientific article itself}

This research was funded by the Deanship of Scientific Research at Princess Nourah bint Abdulrahman University through the Fast-track Research Funding Program.

\section{Creative Commons Attribution}

\section{License 4.0 (Attribution 4.0 International, CC BY 4.0)}

This article is published under the terms of the Creative Commons Attribution License 4.0 https://creativecommons.org/licenses/by/4.0/deed.en US 


\section{Appendix A}

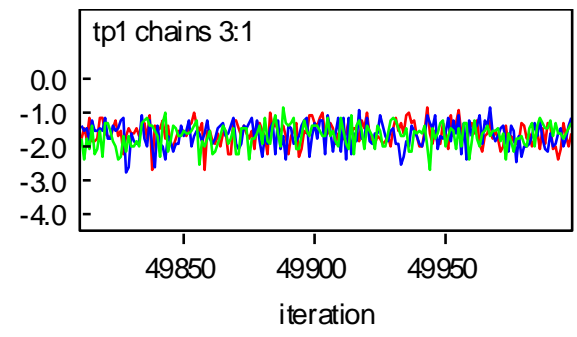

Trace of low stress $\left(\mathrm{t}_{p}\left(\mathrm{x}_{l}\right)\right)$

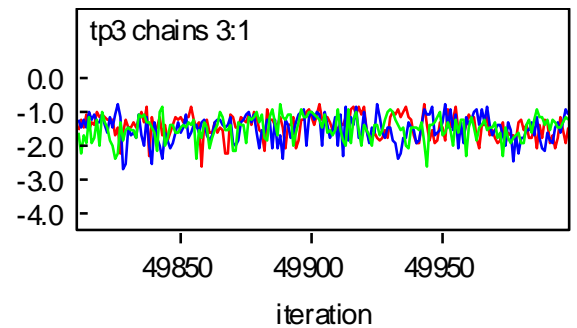

Trace of normal condition $\mathrm{t}_{p}\left(\mathrm{x}_{u}\right)$

Fig. 1 The trace of $\mathrm{t}_{p}\left(\mathrm{x}_{l}\right), \mathrm{t}_{p}\left(\mathrm{x}_{h}\right)$ and $\mathrm{t}_{p}\left(\mathrm{x}_{u}\right)$ for informative prior at $\mathrm{n}=20$
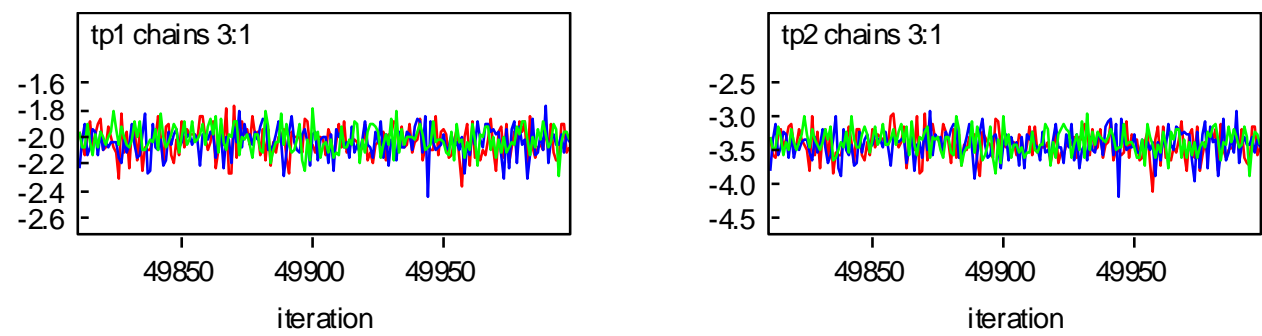

Trace of low stress $\left(\mathrm{t}_{p}\left(\mathrm{x}_{l}\right)\right)$

Trace of high stress $t_{p}\left(\mathrm{x}_{h}\right)$

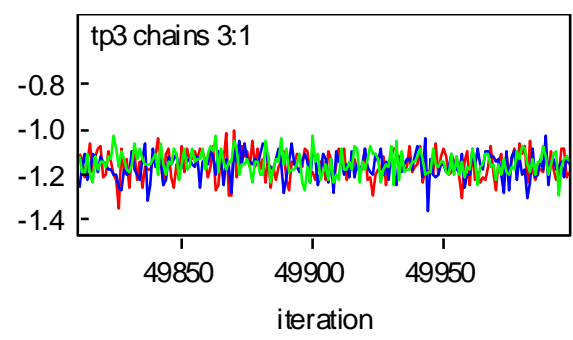

Trace of normal condition $\mathrm{t}_{p}\left(\mathrm{x}_{u}\right)$

Fig. 2 The trace of $\mathrm{t}_{p}\left(\mathrm{x}_{l}\right), \mathrm{t}_{p}\left(\mathrm{x}_{h}\right)$ and $\mathrm{t}_{p}\left(\mathrm{x}_{u}\right)$ for informative prior at $\mathrm{n}=40$ 


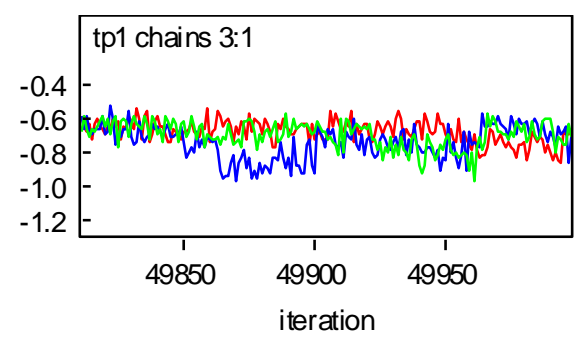

Trace of low stress $\left(\mathrm{t}_{p}\left(\mathrm{x}_{l}\right)\right)$

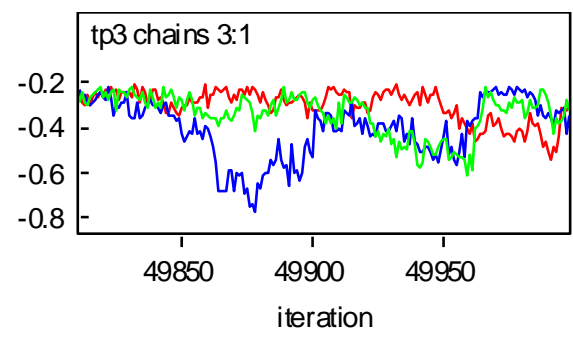

Trace of normal condition $\left(\mathrm{t}_{p}\left(\mathrm{x}_{u}\right)\right)$

Fig. 3 Trace of non-informative prior for $\mathrm{t}_{p}\left(\mathrm{x}_{l}\right), \mathrm{t}_{p}\left(\mathrm{x}_{h}\right)$ and $\mathrm{t}_{p}\left(\mathrm{x}_{u}\right)$ in real data

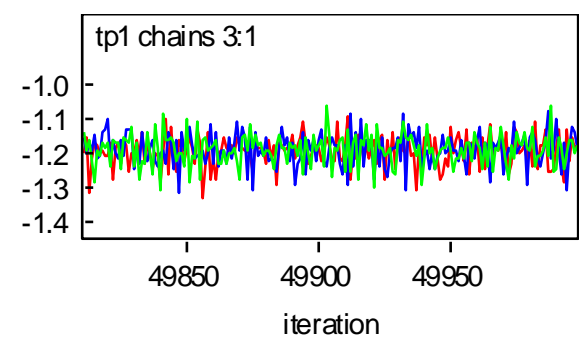

Trace of low stress $\left(\mathrm{t}_{p}\left(\mathrm{x}_{l}\right)\right)$

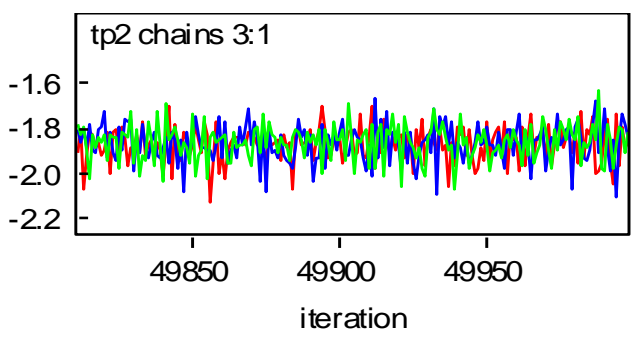

Trace of high stress $\left(\mathrm{t}_{p}\left(\mathrm{x}_{h}\right)\right)$

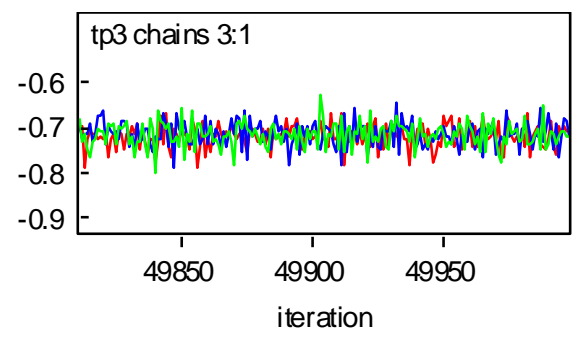

Trace of normal condition $\left(\mathrm{t}_{p}\left(\mathrm{x}_{u}\right)\right)$

Fig. 4 Trace of non-informative prior for $\mathrm{t}_{p}\left(\mathrm{x}_{l}\right), \mathrm{t}_{p}\left(\mathrm{x}_{h}\right)$ and $\mathrm{t}_{p}\left(\mathrm{x}_{u}\right)$ in real data 


\section{Appendix B}

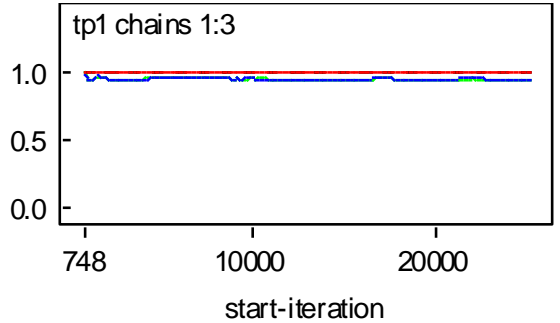

The Gelman Rubin of low stress $\left(\mathrm{t}_{p}\left(\mathrm{x}_{l}\right)\right)$

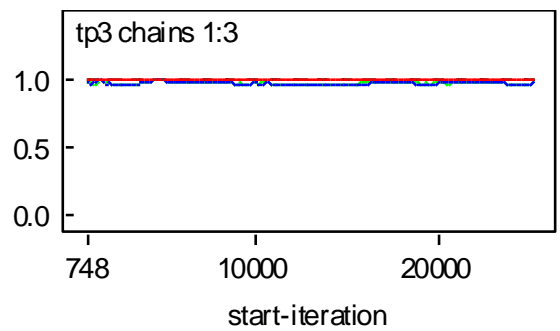

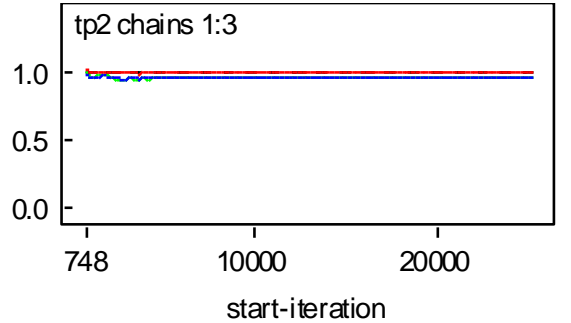

The Gelman Rubin of high stress $\mathrm{t}_{p}\left(\mathrm{x}_{h}\right)$

The Gelman Rubin of normal condition $\mathrm{t}_{p}\left(\mathrm{x}_{u}\right)$

Fig. 5 The Gelman Rubin of $\mathrm{t}_{p}\left(\mathrm{x}_{l}\right), \mathrm{t}_{p}\left(\mathrm{x}_{h}\right)$ and $\mathrm{t}_{p}\left(\mathrm{x}_{u}\right)$, at $\mathrm{n}=20$ with informative prior

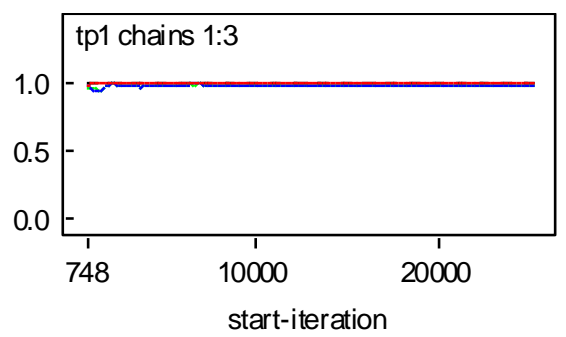

The Gelman Rubin of low stress $\left(\mathrm{t}_{p}\left(\mathrm{x}_{l}\right)\right)$

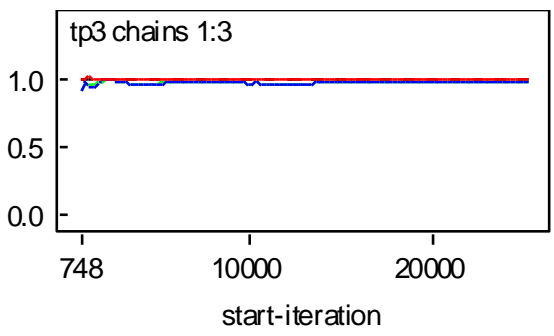

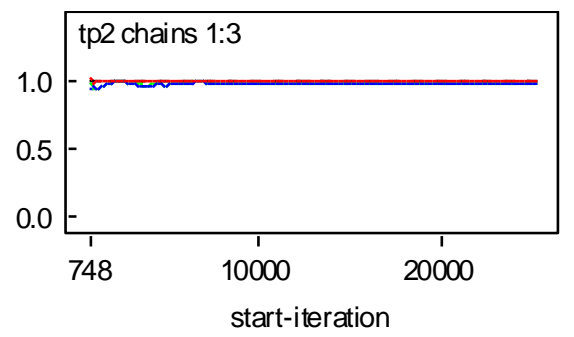

The Gelman Rubin of high stress $\mathrm{t}_{p}\left(\mathrm{x}_{h}\right)$

The Gelman Rubin of normal condition $\mathrm{t}_{p}\left(\mathrm{x}_{u}\right)$

Fig.6 The Gelman Rubin of $\mathrm{t}_{p}\left(\mathrm{x}_{l}\right), \mathrm{t}_{p}\left(\mathrm{x}_{h}\right)$ and $\mathrm{t}_{p}\left(\mathrm{x}_{u}\right)$, at $\mathrm{n}=40$ with informative prior 


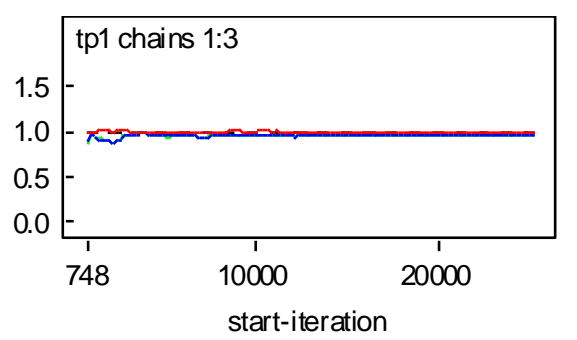

The Gelman Rubin of low stress $\left(\mathrm{t}_{p}\left(\mathrm{x}_{l}\right)\right)$

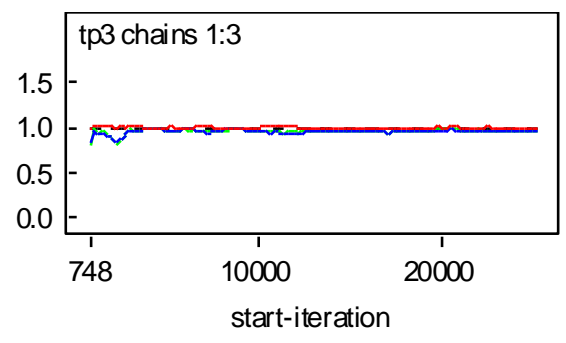

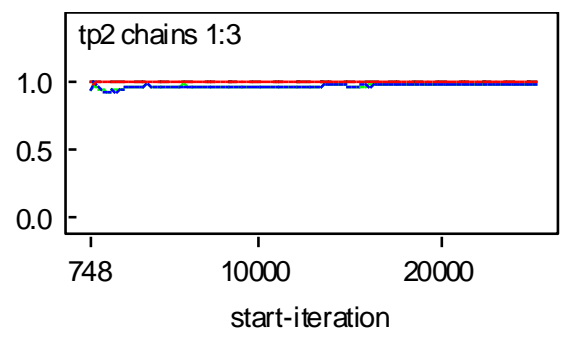

The Gelman Rubin of high stress $t_{p}\left(\mathrm{x}_{h}\right)$

The Gelman Rubin of normal condition $\mathrm{t}_{p}\left(\mathrm{x}_{u}\right)$

Fig. 7 The Gelman Rubin of $\mathrm{t}_{p}\left(\mathrm{x}_{l}\right), \mathrm{t}_{p}\left(\mathrm{x}_{h}\right)$ and $\mathrm{t}_{p}\left(\mathrm{x}_{u}\right)$, in real data with informative prior

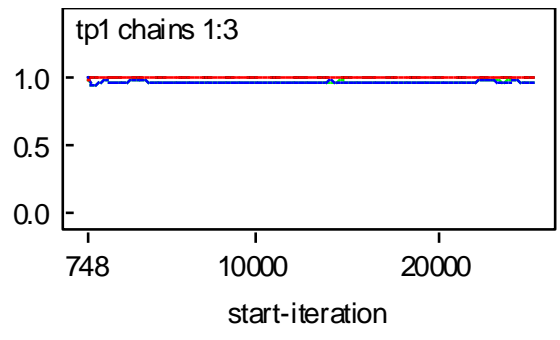

The Gelman Rubin of low stress $\left(\mathrm{t}_{p}\left(\mathrm{x}_{l}\right)\right)$

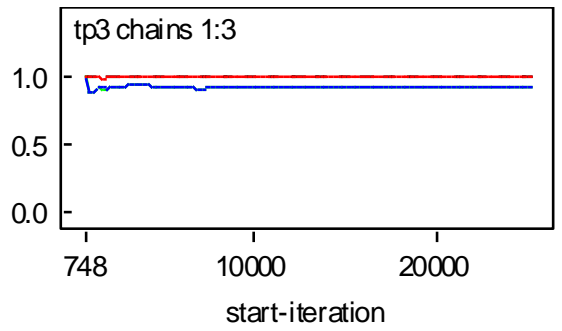

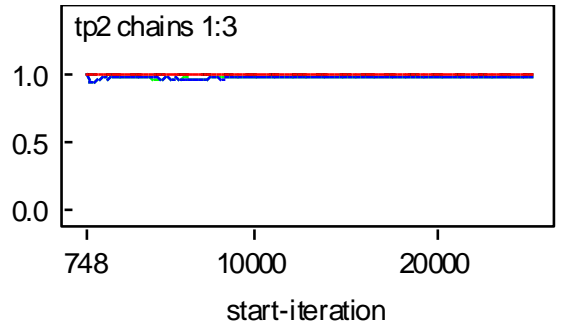

The Gelman Rubin of high stress $t_{p}\left(\mathrm{x}_{h}\right)$

The Gelman Rubin of normal condition $\mathrm{t}_{p}\left(\mathrm{x}_{u}\right)$

Fig. 8 The Gelman Rubin of $\mathrm{t}_{p}\left(\mathrm{x}_{l}\right), \mathrm{t}_{p}\left(\mathrm{x}_{h}\right)$ and $\mathrm{t}_{p}\left(\mathrm{x}_{u}\right)$, in real data with non informative prior 